L. S. Army Missile Command, Physical Sciences Laboratory, Research i Engineering Directorate, Redstone Arsenal, Alabama, and Colorado State Iniversity, Fort Collins, Colorado, U.S.A.

\title{
Power Spectrum, Structure Function, Vertical Wind Shear, and Turbulence in Troposphere and Stratosphere
}

\section{()ikar Essenwanger and Elmar R. Reiter}

With I Figure

Received January 17, 1969

\section{Summary}

It has been shown that a power law exists between vertical velocity differences $T v$ measured over a layer thickness $\Delta h$, of the form $\overline{\Delta v}=a_{0}(\Delta h) a_{1}$. This cxpression may be related directly to a structure function and to a power-specirum function. Statistical derivations of the exponent $a_{1}$ from detailed vertical wind profile measurements show resemblance with the spectral functions obtained for conditions of clear air turbulence.

\section{Zusammenfassung}

'otenzspektrum, Strukturfunktion, vertikale Windscherung und Turbulenz in Troposphäre und Stratosphäre

In früheren Arbeiten wurde gezeigt, daß zwischen den Windgeschwindigkeitsiffcrenzen $\bar{J}$, gemessen über einer Schichtdidke $\Delta h$, ein funktioneller Zusammhing der Art $\overline{\Delta v}=a_{0}(\Delta h) a_{1}$ besteht. Dieser Ausdruck kann mit einer Struk-anktion und einer Spektrum-Funktion in Einklang gebracht werden. Statisti$\therefore$ ! !erleitungen des Exponenten $a_{1}$ aus detaillierten Messungen vertikaler Wind: filc zeigen gute Obereinstimmung mit Spektrum-Funktionen, die unter Cleari.:- Turbulenz-Bedingungen erhalten wurden.

\section{Introduction}

Many investigations of clear air turbulence conducted in recent *an have used power-spectrum analysis (see e. g. ReIter and (1: $\cdots[14,15])$ since TukEY [17] introduced this important mathe- 
matical tool. The utilization of structure functions has been advocated by TATARski [16]. They have, however, rarely been applied to turbulence problems in the free atmosphere. Not much attention has been paid, either, to the structure of wind shears, although close association between wind shears and turbulence exists. It will be shown in this article that knowledge of the structure of wind shears will provide a better understanding of turbulence spectra.

\section{Statistical Wind Shear Relationships}

Essenwanger [5] (see also [7]) demonstrated with the aid of detailed wind soundings that the vertical wind shear is related to the shear interval by a power law as follows:

$$
\overline{\Delta v}=a_{0}(\Delta h)^{a_{1}}
$$

with

$$
\sigma_{\Delta v}=c(\Delta h)^{a_{1}}+A_{0}
$$

and

$$
\sigma_{\Delta v}=A_{0}+A_{1} \overline{\Delta v}
$$

In these equations $\overline{\Delta v}$ denotes the mean total vector shear, expressed in $\mathrm{m} / \mathrm{sec}, \Delta h$ the shear increment, and $\sigma_{\Delta v}$ the standard deviation. $a_{0}, c, A_{0}$, and $A_{1}$ are coefficients or constants, and $a_{1}$ is the exponent of the relationship in question.

As described by ESSENWANGER [5], the exponent $a_{1}$ proved to be 0.5 for mean $\Delta v$, and $1 / 3$ for mean extreme shears. The exponent of 0.5 has also been confirmed by ARMENDARIz and RIDER [1], and by BeLMONT and SHeN [2].

In a subsequent investigation ESSENWANGER [6] showed that the exponent is related to the vertical structure of the wind profile, and that for the mean vector wind shear the vertical persistence (i. e. the meso-structure) causes the exponent to be 0.5. Extreme values of shear appear independent of the bivariate distribution law for zonal and meridional wind shear, and thus the exponent becomes 1/3. Therefore, the conditions for these extreme values may closely resemble the structure of isotropic turbulence.

To gain a better understanding of the relationship between vertical wind structure and the exponent, the following experiment was performed. The wind profile as function of altitude was separated into stationary and non-stationary parts (see EsSENWANGER and BrLlions [8]), and both parts were subjected individually to a wind shear analysis. The resulting distributions are shown in Fig. 1. 
Whereas the smoothed (stationary) part rendered an exponent of $4 / 5$ (Essenwanger and Billions [8]), the exponent was zero for the non-stationary part. According to expression (1) this would mean

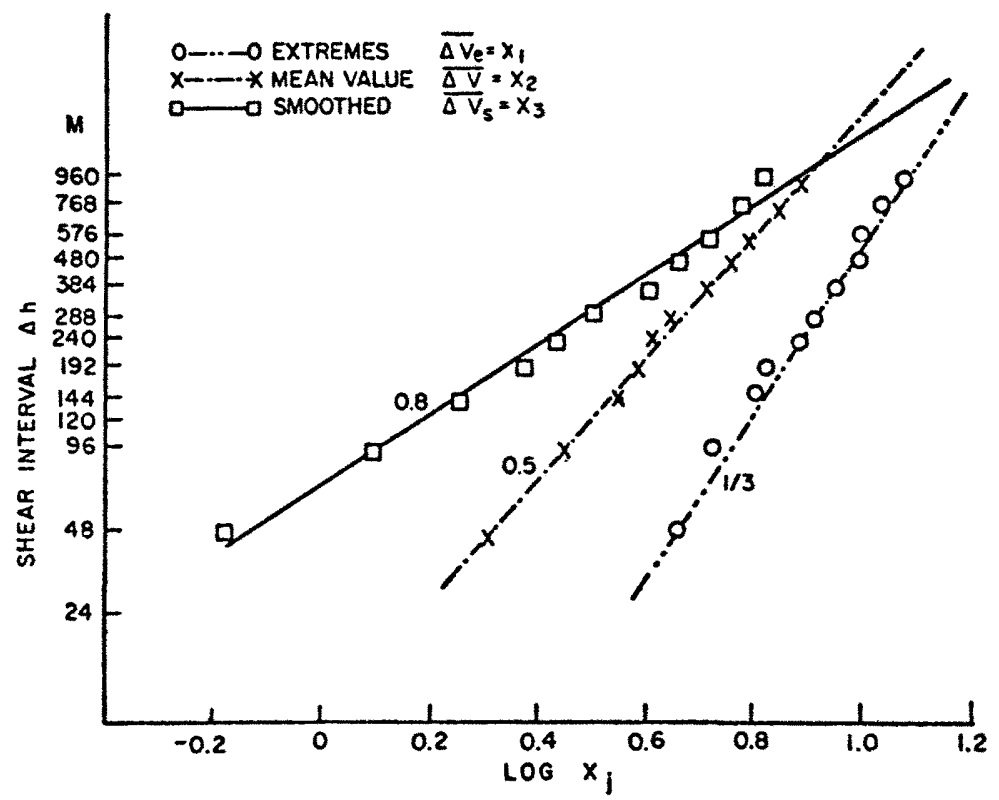

Fig. 1. Relationship Between the Shear Interval $\Delta h$ and the Mean Shear $\Delta v$

that the non-stationary wind fluctuations were completely independent of the thickness of the shearing layer, and scattered about a constant mean value

$$
\overline{\Delta v}=a_{0}
$$

\section{Structure Function and Power Spectrum}

TATARSKI [16] defines the structure function for locally homoreneous and isotropic random fields by

with

$$
D_{f}(r)=\overline{\left[f\left(\vec{r}+\vec{r}_{1}\right)-f\left(\vec{r}_{1}\right)\right]^{2}}
$$

$$
D_{f}(r)=\int_{-\infty}^{\infty}(1-\cos k r) V_{(k)} d k
$$

Where $\vec{r}$ and $\vec{r}_{1}$ are position vectors, and $V_{(k)}$ is a one-dimensional 
spectral density function. If we consider the structure function

$$
D_{f}(r)=c^{2} r^{p} \text { for }(0<p<2)
$$

then the corresponding one-dimensional spectral density function results in

$$
V_{(k)}=\frac{\Gamma(p+1)}{2 \pi} \sin \frac{\pi p}{2} c^{2} k^{b}
$$

where

$$
b=-(p+1)
$$

and $\Gamma$ represents the gamma function.

For $f \equiv v, \vec{r}_{1} \equiv h$, and $\vec{r} \equiv \Delta h$ expression (5) assumes the role of a structure function of the velocity field, the latter given in terms of vertical wind profiles.

\section{Structure Function, Turbulence, and Wind Shear}

It has also been shown by TATARSKI [16] that a locally isotropic random distribution of the wind vector $\vec{v}(r)$ yields nine structure functions of the form

$$
D_{i k}(\vec{r})=\overline{\left[v_{i}\left(\vec{r}+\vec{r}_{1}\right)-v_{i}\left(\vec{r}_{1}\right)\right]\left[v_{k}\left(\vec{r}+\vec{r}_{1}\right)-v_{k}\left(\vec{r}_{1}\right)\right]}
$$

With local isotropy it follows that

$$
D_{i k}(\vec{r})=\left[D_{r r}(r)-D_{t t}(r)\right] n_{i} n_{k}+D_{\imath t} \delta_{i k}
$$

where the $\delta_{i k}$ are Kronecker deltas $\left(\delta_{i k}=1\right.$ for $i=k ; \delta_{i k}=0$ for $i \neq k) ; n_{i}$ are components of the unit vector along $r_{1}$ and

$$
\begin{aligned}
& D_{r r}(\vec{r})=\overline{\left[v_{r}\left(\vec{r}+\vec{r}_{1}\right)-v_{r}\left(\vec{r}_{1}\right)\right]^{2}} \\
& D_{t \prime}(\vec{r})=\overline{\left[v_{t}\left(\vec{r}+\vec{r}_{1}\right)-v_{t}\left(\vec{r}_{1}\right)\right]^{2}}
\end{aligned}
$$

are longitudinal and transverse structure functions. $v_{r}$ are the velocity components parallel to the vector $r_{1}, v_{t}$ the components normal to $\vec{r}_{1}$.

For incompressible fluids ( $\nu \ll c$, where $c$ is the velocity of sound) Tatarski [16] derives

$$
D_{t t}(\dot{r})=\frac{1}{2 r} \frac{d}{d r}\left(r^{2} D_{r r}\right)
$$


According to Kolmogorov [11] and Oвukrov [12] turbulence in the (isotropic) inertial subrange follows the "two-thirds law"

$$
D_{r r}(\vec{r})=C(\varepsilon r)^{2 !} \cdot \text { for } l_{0}<<r<<L
$$

where $C$ is a dimensionless constant of the order of unity, $\varepsilon$ is the rate of dissipation of kinetic energy, and $l_{0}$ and $L$ are the inner and outer scales of turbulence. It follows from (13) that

$$
D_{\| l}(\vec{r})=\frac{4}{3} C(\varepsilon r)^{2 / s} \text { for } l_{0}<<r<<L
$$

A comparison between expression (1) and (12) shows that the vertical shear of the horizontal wind vector relates to the transverse structure function as

$$
\overline{\Delta v}=\sqrt{D_{t t(\Delta h)}}
$$

or. for the inertial subrange of turbulence

$$
a_{0}(\Delta h)^{a_{1}}=\sqrt{\frac{4}{3} C^{1}} \cdot \varepsilon^{1 / s}(\Delta h)^{1 / s}
$$

From this it follows that

$$
a_{1}=1 / 3 \text { and } 2 a_{1}=p=2 / 3
$$

which ESSENwanger [5] found to hold for mean extreme shears. $t_{0}$ turns out to be a function of the rate of dissipation, $\varepsilon$. At the same time. because of (7) and (8), velocity spectra follow the well-known -53 law"

$$
V(k) \propto \cdot k^{-s / 4}
$$

The exponent for mean shears was reported by EssENwanger to $\because u_{1}=1 / 2$. From (16) and (7) it follows that $p=1$, and from (9) $\therefore$. the "spectrum slope" should be $b=-2$. It has been mentioned culier that these mean shears contain turbulence as well as meso$\because$ uture. It has been shown by Hines [10] and by Weinstein et al. $\because$ that the meso-structure present in vertical wind profiles above $\therefore$ tropopause may be explained by the effect of internal gravity (inertial waves) having horizontal wavelengths of $10^{2}$ to 
$10^{3} \mathrm{~km}$. Phillips [13] considered the spectra of internal waves and arrived at the striking result that

$$
V(k, \Delta h) \propto k^{-2}
$$

for $K \cdot \Delta h \ll 1$, where $\Delta h$ characterizes the thickness of the layer with strong stratification. This condition is certainly met with internal waves described by Hines [10]. Thus, Phillips' theoretical derivations are in excellent agreement with data obtained from detailed vertical wind profiles.

The non-stationary wind profiles, found by EsSENwANGER and Billions [9] to yield an exponent $a_{1}=0$ [see Eq. (4)], lead to $p=0$ and hence a "white noise" spectrum with a slope $b=-1$.

The smoothed (stationary) part of vertical wind profiles, according to EsSENwANGER and Billions [8] which contained the persistent meso-scale of atmospheric structure, yielded $a_{1}=4 / 5$. This results in $p=8 / 5$ for the structure function, and $b=-13 / 5$ for the spectrum slope. This value lies between $b=-11 / 5$ derived by BoLGIANo $[3,4]$ for the "buoyant subrange", and $b=-3$ found by VINNICHENko et al. [18] to hold for spectra measured in horizontal flight by aircraft at wave length along the flight path of $\lambda_{x}>600$ to $800 \mathrm{~m}$.

A linear trend in wind shears may be expressed by Eq. (1), with $a_{1}=1$. Such a trend will yield a spectrum slope of $b=-3$, if one extended the condition imposed upon Eq. (7) to $p=2$. Hence, one may speculate to what extent VinNichenko's et al. [18] measurements were influenced by a linear trend.

\section{Conclusion}

Even though the comparisons between vertical wind shears, structure functions, and power spectra which have been presented in the foregoing discussion have been made for transverse velocity fluctuations $(\overline{\Delta v} \perp \overline{\Delta h})$ similar reasoning may be applied to longitudinal fluctuations $(\overline{\Delta v} \| \overline{\Delta x})$ as they may be obtained, for instance, from aircraft records [see Eq. (12) through (15)]. The same exponential. relationship should hold as derived above, only constant coefficients [ $a_{0}$ in Eq. (1)] should differ by a factor of $3 / 4$ [see Eq. (15)]. One may, thus, avoid the computation of spectra from the Fourier transformation of autocorrelation functions by resorting to a simple treatment of vertical and/or horizontal wind shears as outlined by Eq. (1). The relationship between wind shears and power spectra, demon- 
iratco by TATARski's [16] structure function; also simplifies the interpretation of turbulence data.

From the foregoing it appears that the relation between $\Delta v$ and $1 / h$ is limited by "white noise" conditions $\left(a_{1}=0\right)$ on the one hand, and by a linear trend without turbulence or mesostructure $\left(a_{1}=1\right)$ on the other hand.

\section{References}

Irnexpariz, M., and L. J. Rider: Wind Shear for Small Thidkness Layers. J. Applied Meteorol. 5, 810-815 (1966).

: Bitmove. A. D., and W. C. Shen: Comparison of Jimsphere and Rawinsonde Wind Shears. Control Data Corporation, Final Report, Contract N 00014-66(O) 12i, to Advanced Research Project Agency, Washington, D. C., 1966.

: Butisino. R. JR.: Turbulent Spectra in a Stable Stratified Atmosphere. 1. (icoph. Res. 64, 2226 ff. (1959).

: Bminiso. R. JR.: Structure of Turbulence in Stratified Media. J. Geophys. Kis. 67. 3015-3024 (1962).

Finwasger, O. M.: On the Derivation of Frequency Distributions of Vector Shicur Values for Small Shear Intervals. Geofis. pura appl. 56, 216-224 1(mi3).

Kwrwwixgr. O. M.: Statistical Parameters and Percentile Values for Vector Wind Shear Distributions of Small Increments. Arch. Met. Geoph. Biokl., A, 15. ill-61 (1965).

- Entrwanger, O. M.: Comments on "Mesoscale Structure of $11-20 \mathrm{~km}$ Winds". J. Appl. Meteor 6, 591-593 (1967).

- Enriwinger, O. M., and N. S. Brllons: The Stationary and Nonstationary "Iind Profile. Pure and Applied Geophysics 60, 160-166 (1965).

Ewinwaver, O. M., and N. S. Bullons: On Wind Shear Distributions for imaller Shear Intervals. U.S. Army Missile Command Report No. RR-TRii.t. 1965.

"H、rs. C. O.: Internal Atmospheric Gravity Waves at Ionospheric Heights. C.anadian Journal of Physics 38, 1441-1481 (1960).

Kinsmcorov, A. N.: Dissipation of Energy in Locally Isotropic Turbulence. 1).klady Akad. Nauk SSSR, 32 (1941). German translation in: Sammelband ar Stutistischen Theorie der Turbulenz, Akademie-Verlag, Berlin, p. 77 (1958).

1) knos. A. M.: On the Distribution of Energy in the Spectrum of Turbu... llow. Doklady Akad. Nauk SSSR, 32 (1941).

"uirs. O. M.: The Generation of Clear Air Turbulence by the Degradation - Internal Gravity Waves. Proc. Internat. Colloquium on Atmospheric Tur$\therefore$ nce and Radio Wave Propagation, Moscow, 1965, pp. 130-138 (1967). : . E. R.. and A. Burns: The Structure of Clear-Air Turbulence Derived TOPCAT Aircraft Measurements. J. Atm. Sci. 23, 206-212 (1966). $\therefore:$ \&. E. R.. and A. Burns: Atmospheric Structure and Clear-Air Turbu$\because \quad$ lroc. Internat. Colloquium on Atmospheric Turbulence and Radio $\therefore$ Prepagation, Moscow, 1965, pp. 53-64 (1967).

in. I. I.: Wave Propagation in a Turbulent Medium. New York: 
17. TukeY, J. W.: The Sampling Theory of Power Spectrum Estimates in Symp. on Application of Autocorrelation Analysis to Physical Problems, Woods Hole, 1949; Office Naval Research, NAVEXOS-P-735 (1949).

18. Vinnichenko, N. K., N. Z. Pinus, and G. N. Shur: Some Results of the Experimental Turbulence Investigations in the Troposphere. Proc. Internat. Colloquium on Atmospheric Turbulence and Radio Wave Propagation, Moscow, 1965, pp. 74-75 (1967).

19. Weinstein, A. I., E. R. Reiter, and J. R. Scoggins: Mesoscale Structure of $11-20 \mathrm{~km}$ Winds. J. Appl. Meteorol. 5, 49-57 (1966).

Authors' addresses: Prof. Dr. O. Essenwanger, 610 Mountain Gap Drive, Huntsville, AL 35803, U.S. A. - Prof. Dr. E. R. Rerter, 1513 Hillside Drive, Fort Collins, CO 80521 , U. S. A. 